\title{
Polishing of Chemical Oxygen Demand (COD) Using Moving Bed Bio- Reactor
}

\author{
Jamal Ali Kawan ${ }^{1, a^{*}}$, Rakmi Abd-Rahman ${ }^{1, b}$, Othman Bin Jaafar ${ }^{2, c}$ \\ and Fatihah Suja ${ }^{2, d}$ \\ ${ }^{1}$ Department of Chemical and Process Engineering, Faculty of Engineering \\ Universiti Kebangsaan Malaysia (UKM), 43650 Bangi, Selangor, Malaysia \\ 2 Department of Civil Engineering, Faculty of Engineering \\ Universiti Kebangsaan Malaysia (UKM), 43650 Bangi, Selangor, Malaysia \\ ajamal_kawan@yahoo.ie, ${ }^{b}$ rakminuraini@yahoo.com, 'ojaafar@gmail.com, \\ fatihahfkab@gmail.com
}

Keywords: Effluent polishing; Moving bed biofilm reactor; Biofilm carriers; Plastic media; Enviro Multi Media; COD removal.

\begin{abstract}
The pilot-scale experiment in moving bed biofilm reactor (MBBR) with a capacity of 433 L was carried out for this study with real life situations, it was decided that the complete research work must be done under as realistic conditions as possible, i.e. with real municipal wastewater, chemical free and with local commercially available products such as carriers for biofilm reactor. The reactor was start-up in 30/9/2013 up to date, Effluent from clarifier of STP used as influence of MBBR for polishing. MBBR is using continues down flow to polish effluent municipal wastewater from a faculty of new building engineering community in UKM to get the water free from main pollutant parameters, for reuse in the irrigation or discharge to the river. Laboratory experiments will conduct with different hydraulic retention time (HRT), filling ratio of plastic (Enviro Multi Media) in the MBBR about 5\%. Aerobic reactors used the majority of the decaying organic material. An average removal rate of $41.75 \%, 32.85 \%, 24.80 \%$ and $35.77 \%$ of initial chemical oxygen demand (COD) were achieved under a HRT of $24 \mathrm{hr}, 12 \mathrm{hr}, 6 \mathrm{hr}$ and $2 \mathrm{hr}$, respectively. The model simulated results showed good agreements with experimental results. The model could be employed in the design of a full-scale MBBR process for simultaneous removal of organic carbon from effluent STP.
\end{abstract}

\section{Introduction}

To improve the quality of water, a water polishing treatment is used in order to reduce the concentrations of the pollutant parameters at the required level for reuse or final destination. Water polishing refers to the process by which waters that goes through additional treatment, removing things that are difficult to remove. This mechanism has the ability to guarantee the preservation of aqueous life and water resources for the future.

A relatively new advancement in the field of fixed film biological treatment system is the moving bed biofilm reactor (MBBR), a process developed in the 1980s and early 1990s [1]. Since then MBBR has evolved to being a common process used worldwide as an integral part of treatment plant or as an added treatment unit.

The basis of MBBR process is the conventional activated sludge and biofilter processes. MBBR also takes advantage of the activated sludge process and conventional fixed film systems, without their disadvantages. The MBBR reactor volume has no unused space, an advantage of having the biofilm reactors totally mixed. MBBR has a great advantage over the activate sludge process as MBBR does not require sludge recirculation nor a large sedimentation tank [2, 3].

The MBBR process has many additional advantages $[4,5,6]$ : 
- Simplicity compared to activated sludge.

- No need for returning settled sludge to the reactor even at short hydraulic retention times.

- Controlled biomass separation and more advanced nutrient removal ability.

- No backwash requirement.

- The reactors contain a high concentration of microorganisms enabling rapid degradation rates and thus small reactor sizes (reduced footprint).

- Without a threat to clogging.

\section{Biofilm Carrier}

Biofilm carriers have a large surface area with a density $\pm 1.0 \mathrm{~kg} / \mathrm{m}^{3}$; and are made from high density polyethylene or polypropylene [7,8]. Another advantage of MBBR arises here; its filling fraction in the reactor is subject to preferences. Recommended filling fractions should be less than $70 \%$ so that the biofilm carrier suspension can move freely [9]. Like the activated sludge system, MBBR uses the whole reactor, but does not require sludge cycle.

The microscale MBBR processes involved in converting unwanted pollutants are complicated. This makes it difficult to develop accurate mathematical processes, optimizing performance or identifying important variables.

\section{Materials and Methods}

The STP locates inside UKM besides new engineering building. The waste water from new engineering building flows into STP through the mechanical screen, the treated water is discharged into the downstream of Langat River.

This study aims to fix Moving Bed Bio Reactor (MBBR) on the effluent of clarifier, and analyze the influent and effluent of the MBBR; the parameters will experiment in the chemical/civil engineering laboratory to know the efficiency of MBBR and the ability to remove COD. A schematic diagram of the system is given in Figure 1.

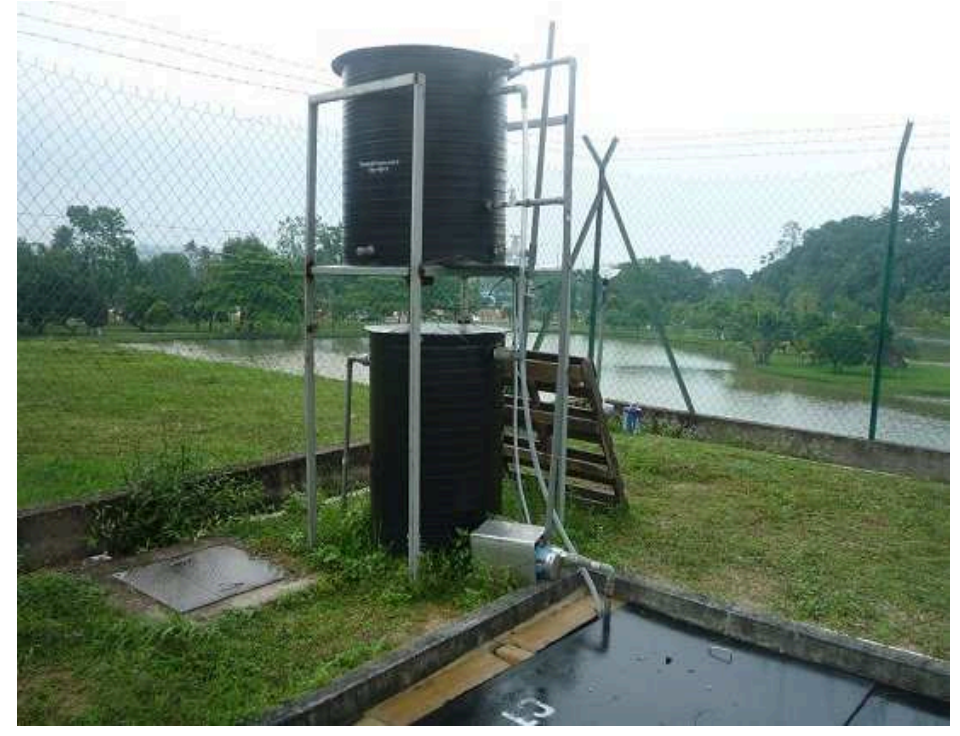

Fig. 1 Moving bed biofilm reactor (MBBR) used during pilot scale

Wastewater samples will be collected from an influent and effluent of moving bed bioreactor during the operation period. Two samples will be collected 10.00 am to $11.00 \mathrm{am}$, three times a week with different Hydraulic Retention Time (HRT) from October 2013 to October 2014. All 
samples will be collected in sterile $1000 \mathrm{ml}$ polyethylene bottles, which has soaked and clean before the sampling. The fresh sample process at the lab directly.

\section{COD Removal with Various HRT}

COD is often measured as a rapid indicator of organic pollutant in water and gives an indication of the efficiency of the treatment process. COD is measured on both influent and effluent water according to the standard methods [10]. The efficiency of the treatment process is normally expressed as COD Removal, measured as a percentage of the organic matter purified during the cycle. The scope of the study was initially limited to investigate how effluent biological polishing of COD could be achieved in a MBBR process.

Effluent from classifier used as influence of MBBR for polishing, start with different HRT with simple calculations HRT=V/Q. Started with $H R T=24 \mathrm{hrs}$ for 3 months to grow a biofilm on plastic media, then run at HRT $=12 \mathrm{hr}, 6 \mathrm{hr}$ and $2 \mathrm{hrs}$; refer to table 1 . During these HRT measure $\mathrm{pH}$ and DO in the reactor and IN and OUT stream for COD to get removal efficiency for biofilm process.

Table 1, Hydraulic Retention Time (HRT), and flow rate (Q)

\begin{tabular}{ccccc}
\hline Volume [L] & 433 & 433 & 433 & 433 \\
\hline HRT [hours] & 24 & 12 & 6 & 2 \\
Flow rate [L/hr] & 18.04 & 36.08 & 72.17 & 216.5 \\
\hline
\end{tabular}

\section{Results and Discussion}

The daily variation of influent and effluent concentration of chemical oxygen demand was studied during the operation period. From HRT $=24 \mathrm{hrs}$ in the Figure. 2 the range of influent and effluent concentration of COD was 28 to $8 \mathrm{mg} / \mathrm{L}$ and 18 to $3 \mathrm{mg} / \mathrm{L}$, respectively, where the average COD removal was $41.75 \%$ as shown in figure 3. The maximum removal of COD was $72.72 \%$ during this study period.

As illustrated in the HRT $=12 \mathrm{hrs}$ and HRT $=6 \mathrm{hrs}$ in the figure 2 and figure 3 the range of influent and effluent COD concentration was 18 to $6 \mathrm{mg} / \mathrm{L}, 31$ to $17 \mathrm{mg} / \mathrm{L}$ and 14 to $4 \mathrm{mg} / \mathrm{L}, 27$ to $2.5 \mathrm{mg} / \mathrm{L}$ where the average removal was $32.85 \%, 24.8 \%$, respectively. The maximum removal was $86.84 \%$ of the 6 hours HRT during this study. From the part of HRT=2 hrs the range of influent and effluent concentration of COD was 21 to $10 \mathrm{mg} / \mathrm{L}$ and 13 to $7 \mathrm{mg} / \mathrm{L}$, respectively, where the average COD removal was $35.77 \%$.

Of all the periods of HRT=24 HR, $12 \mathrm{hrs}, 6 \mathrm{hrs}$ and $2 \mathrm{hrs}$ in (figure 2) the range of influent and effluent concentration of COD was 34 to $6 \mathrm{mg} / \mathrm{L}$ and 27 to $2.5 \mathrm{mg} / \mathrm{L}$, respectively, where the average COD removal was $33.79 \%$. The minimum and maximum COD removal was $4.76 \%$ to $54.55 \%$ at all study periods HRTs.

Ultimately, it was demonstrated that The average COD removal efficiency at 24, 12,6 hrs HRTs were gradually declined $(41.75 \%, 32.85 \%$ and $24.8 \%$, respectively), while the removal efficiency was again increased to $35.77 \%$ at the HRT of $2 \mathrm{hrs}$, because of well mixing with the down flow liquid velocity, enhancing growth of a thin layer of biomass in the carriers and improved the organic removal efficiency. 


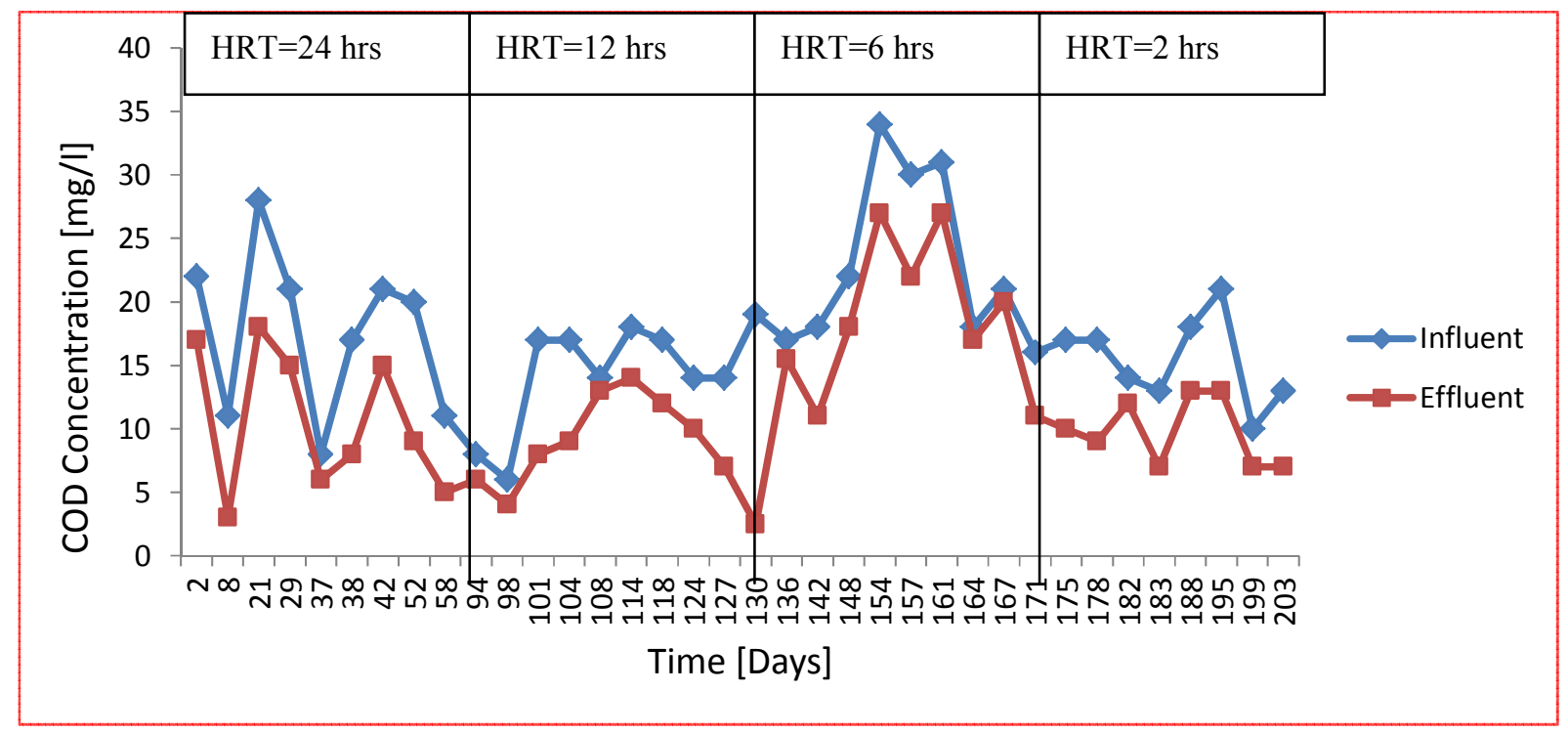

Fig. 2 Influent and Effluent concentrations versus time using MBBR (5\%)

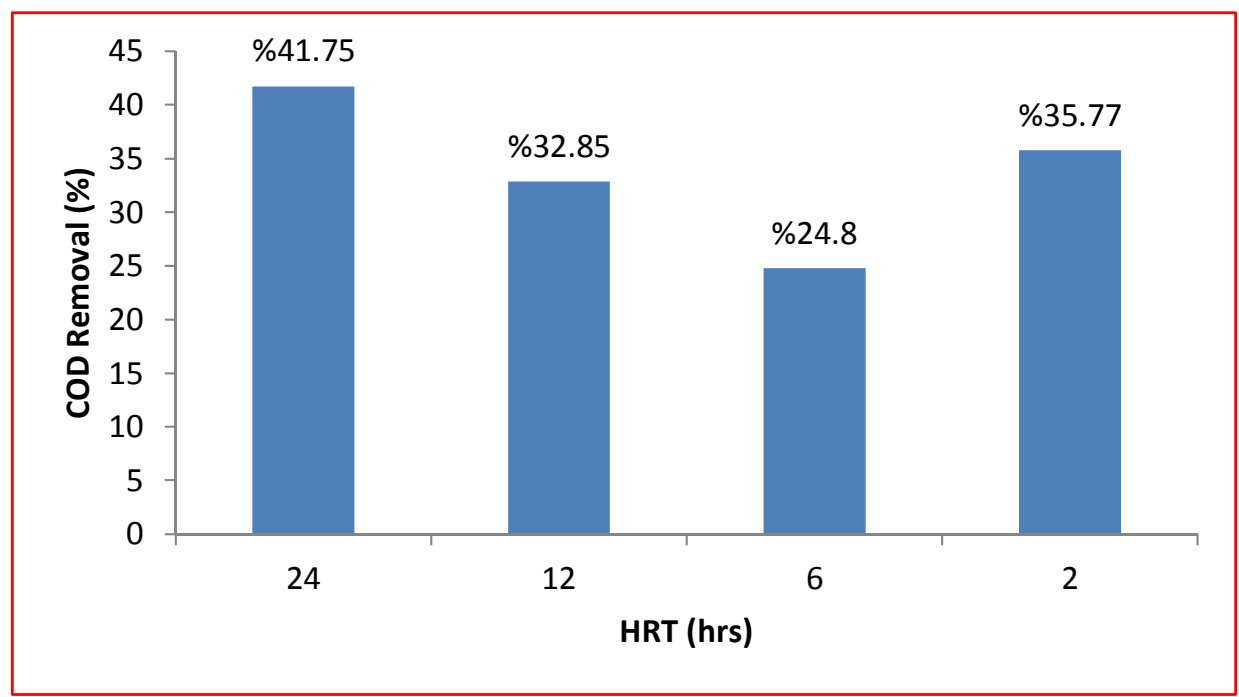

Fig. 3 The average removal of COD for different Hydraulic Retention Time (HRT)

\section{Conclusion}

In this study is focused on the effluent polishing of COD using Moving Bed Biofilm Reactors (MBBRs), where the effluent is used from STP new building of engineering in UKM Bangi and the main treatments performed is the elimination of total organic matter (COD). The results will show that the moving biofilm is effective in the removal of COD, and this study will be carried for 12 months in various HRT.

\section{Acknowledgements}

The authors are thankful to UKM Bangi for providing the analytical and logical support in order to publish the findings of these studies. 


\section{References}

[1] Ødegaard, H. (2006) Innovations in wastewater treatment: the moving bed biofilm process. Water Science and Technology. 53 (9) 17-33.

[2] M. Rodgers and X.-M. Zhan. Moving-medium biofilm reactors. Rev. Environ. Sci. Biotechnol.,2(2-4):213-224, 2003.

[3] Xiao, G. Y., and J. Ganczarczyk. 2006. Structural features of biomass in a hybrid MBBR reactor. Environmental Technology 27, (3): 289-98.

[4] Rusten, B. and Neu, K. E. (1999) "Down to Size", Water Environment \& Technology, 11(1), 27-34 pp.

[5] Ødegaard, H., Ruaten, B. and Westrum, T. (1994): A New Moving Bed Biofilm ReactorApplications and Results", Water Science Technology, 29(10-11), 157-165 pp.

[6] Andrettola, G.; Foladori, P.; Gatti, G.; Nardelli, P.; Pettena, M. and Ragazzi, M. (2003) "Upgrading of a Small Overload Activated Sludge Plant Using a MBBR System",Journal of Environmental Science and Health, A38(10), 2317-2338 pp.

[7] Ødegaard, H., Ruaten, B. and Westrum, T. (1994): “A New Moving Bed Biofilm ReactorApplications and Results", Water Science Technology, 29(10-11), 157-165 pp.

[8] Delenfort, E., and Thulin, P., (1997): "The use of Kaldnes suspended carrier procces in treatment of wastewaters from the forest industry", Water Science Technology, 35(2-3):123-130.

[9] Rusten, B., Eikebrokk, B., Ulgenes, Y., and Lyrgen, E. (2006) Design and operation of the Kaldnes moving bed biofilm reactors. Aquaculture Engineering. 34, 322-331.

[10] APHA (1995). Standard methods for the examination of water and wastewater. Washington, DC, American Public Health Association. 